\title{
Lappische Lehnwörter im ältesten Einödgebiet Finnlands
}

1. Die Verbreitung der lappischen Bevölkerung in Finnland hat die Forscher schon lange beschäftigt. In Ermangelung schriftlicher Zeugnisse aus älteren Zeiten hat man verschiedene Typen sprachlichen Materials analysiert, z.B. Ortsnamen. ${ }^{1}$ Finnland verfügt über eine grosse Anzahl Ortsnamen auf Lappi-, Poro-, Kota- usw., von denen die meisten sicher auf eine mehr oder minder feste lappische Besiedlung hinweisen. In den südwestlichen Teilen des Landes sind sie allerdings nicht so geläufig wie in jenen Gegenden, in denen sich die älteste feste Besiedlung befand, was wir aufgrund von archäologischen Funden und anderen älteren Angaben wissen.

Vergleichen wir die Ausbreitung der eisenzeitlichen Besiedlung in Südwestfinnland z.B. mit den Lappi-Namen ${ }^{2}$, welches der wichtigste Namenstypus ist, können wir eine Vorstellung über die Voraussetzungen für die Namengebung und die Funk-

' K. B. Wiklund, Lapparnas forna utbredning i Finland och Ryssland, belyst af ortnamnen. Le Monde Oriental, 5, 1911. Jalo Kalima, Lappalaisten muinainen levintä Venäjällä ja Suomessa paikannimien valossa. Virittäjä 1912. T. I. Itkonen, Lappalaisperäisiä paikannimiä suomenkielen alueella. Virittäjä 1920 und Lisiä Keski-ja Etelä-Suomen lappalaisperäiseen paikannimistöön. Virittäjä 1926. Bror Åkerblom, Lapp-namnen i Finland, Folkmålsstudier XI, Helsingfors 1945. Verf. führt alternative Deutungen der Lappi-Namen an und zweifelt an einer in älterer Zeit im südwestlichen Finnland vorhandenen festen lappischen Besiedlung. T. I. Itkonen, Lapparnas förekomst i Finland, Ymer 67. Stockholm 1947. T. I. Itkonen, Suomen lappalaiset vuoteen 1945 I. Porvoo-Helsinki 1948 S. 97-. Armas Luukko, Etelä-Pohjanmaan historia II. Keskiaika ja 1500-luku. Helsinki 1950 S. 14.

${ }^{2}$ S. die Karte: T. I. Itkonen 1948 I, S. 103. 
tion des Namens bekommen. Dieser Namenstypus fehlt fast völlig im Flusstal des Kokemäenjoki, in der Umgebung von Kyrösjärvi und in den zentralen Teilen von Häme. ${ }^{3}$ Dagegen begegnen viele dieser Namen in der nahen Umgebung der genannten Gegenden und besonders dicht in den äusseren Kirchspielen des alten Kyrö (nördlichster Teil von Obersatakunta), in den Gebieten um den Fluss Kyröjoki im südlichen Ostbottnien, im Nordosten von Häme und sogar in den zentralen Teilen des heutigen Savo.

Die südlichsten Lappi-Namen finden wir u.a. im Flusstal des Loimijoki und in der südwestlichsten Ecke von Häme. Sie bilden ferner einen Gürtel in den innersten Teilen von Uusimaa. Vergleichen wir dieses Ergebnis mit einer Karte über die Ausbreitung der festen Besiedlung zu Beginn der Neuzeit, können wir nicht ausschliessen, dass ein Zusammenhang besteht zwischen dem Fehlen einer festen Besiedlung und dem Vorkommen der Lappi-Namen. ${ }^{4}$

Wie erklären wir aber die weissen Stellen auf der Ortsnamenkarte? Für die Provinz Varsinais-Suomi gilt wohl, dass es dort nur wenige Lappen gab und dass diese auf jeden Fall nicht sesshaft waren. ${ }^{5}$ Das Fehlen solcher Namen in Gegenden mit

${ }^{3}$ Jalmari Jaakkola, Suomen varhaishistoria. Porvoo 1956 S. 21, 271-282. Helmer Salmo, Satakunnan historia II. Vammala 1952 S. 136-138, 444-449. Ella Kivikoski, Hämeen historia I. Hämeen rautakausi. Hämeenlinna $1955 \mathrm{~S}$. 44-48.

4 Armas Luukko, Suomen asuttaminen. Oma maa 1. Porvoo-Helsinki 1958 S. 11. S. die Karte: Toivo Vuorela, Suomalainen kansankulttuuri. Porvoo 1975 S. 22. Die finnischen hiisi-Namen kommen in einem ganz anderen Gebiet vor als die Lappi-Namen. Sie konzentrieren sich auf die am dichtesten besiedelten Gegenden von Südwestfinnland und füllen die weissen Stellen auf der Karte über die Lappi-Namen aus. (Mauno Koski, Itämerensuomalaisten kielten Hiisi-sanue. Turku 1967 S. 179-180.) Vgl. Asbjörn Nesheim, Finnish hiisi and Lappish sii'dâ. FUF 30: 3. Bergsland lässt die Frage offen, ob es sich bei dem Wort um eine frühe Entlehnung aus dem Finnischen ins Lappische handelt (Knut Bergsland, Suomen hiisi. Virittäjä 1964 S. 247). Verbreitung und Frequenz des hiisi-Namens lassen es zu, ihn am ehesten mit der ältesten finnischen Besiedlung Südwestfinnlands zu verbinden.

5 Vgl. Erkki Itkonen, Lappalaista merkitsevistä nimityksistä ja niiden historiallisesta taustasta. Virittäjä 1941 S. 84. 
alter Besiedlung kann am leichtesten unter Berücksichtigung der Funktion dieses Ortsnamentyps erklärt werden. Er markiert ja den Unterschied zwischen der fest ansässigen und kulturell dominierenden Person gegenüber dem oft unterworfenen und ausgenutzten Individuum. ${ }^{6}$

Als die Einödfahrten an Intensität zunahmen, entstand eine günstige Voraussetzung für die Namengebung. Diese Namen kamen in Gegenden zur Anwendung, wo sich die verschiedenen Bevölkerungsgruppen trafen, d.h. in einem gewissen Abstand von der eisenzeitlichen Besiedlung. Die grosse Anzahl von Lappi-Namen an der Peripherie des ältesten Besiedlungsgebietes zeigt uns deshalb, wo die Kontakte am intensivsten waren. In den übrigen Teilen des Landes finden wir die lappischen Ortsnamen gleichmässig verteilt. Hier spiegeln sich die kontinuierlichen finnisch-lappischen Beziehungen wider, die allmählich dazu führten, dass sich die Lappen assimilierten oder gezwungen wurden, sich nach Norden zurückzuziehen.

Voionmaa äussert ähnliche Gedanken im Zusammenhang mit seiner Untersuchung der Lappi-Namen in der Provinz Uusimaa. "Vorzugsweise gruppieren sie sich in den unbesiedelten äusseren und mittleren Gebieten des historischen Uusimaa." 7 Er verknüpft auch die Lappi-Namen mit der frühen Häme-Expansion aus dem alten Vanaja und Hauho in Richtung Finnischer Meerbusen und Ladogasee. Konzentriert ist das Vorkommen der Namen bis zu den nördlichen Teilen von Mittel-Uusimaa und im Süden und Südosten des Päijänne, wohin die Einödfahrten gingen. ${ }^{8}$

Wenn die historischen Quellen die Lappen im südlichen Finnland auch erwähnen, sind die Angaben doch recht dürftig. Kurze Notizen finden sich u.a. von den Kirchspielen Sääksmäki

\footnotetext{
6 Julku meint, in den lappischen Ortsnamen eine "Front" und eine "Linie" unterscheiden zu können; er konzentriert sich auf die Nordgrenze der Besiedlüng in der Übergangsperiode von der vorhistorischen zur historischen Zeit. Kyösti Julku, Suomen saamelaisen asutuksen vaiheet ("Pohjola 2000" -seminaari 5-8. 8. 1968. Esitelmät ja alustukset). Oulu 1969, S. 170.

7 Väinö Voionmaa, Uudenmaan lappalaiset. Suomalaisen Tiedeakatemian Esitelmät ja Pöytäkirjat 1943. Helsinki 1944, S. 88.

8 J. Jaakkola 1956 S. 207-210.
} 
(1390) ${ }^{9}$ und - hauptsächlich in Überlieferungen aus Vesilahti, Lappi (Bez. Turku), Janakkala, Tottijärvi. ${ }^{10}$ Im nördlichen Häme und in den Grenzgebieten zwischen Satakunta und Südostbottnien ist bedeutend mehr überliefert. In diesen nördlichen Gebieten gibt es noch immer volkstümliche Traditionen über die Lappen und ihre Lebensverhältnisse, auch wenn diese Überlieferungen heute nur noch recht vage sind. Es scheint, als hätten sich diese Traditionen in Parkano und Kihniö in Obersatakunta am besten bewahrt. ${ }^{11}$ Sagen und verschiedene Typen von Sprichwörtern und Redensarten im Zusammenhang mit den Lappen und mit Angaben über lappische Wohnorte sind aus dem sog. "Lappland von Satakunta" erhalten. ${ }^{12}$

Die Grenzbezirke von Obersatakunta und Südostbottnien mit ihren ausgedehnten Heide- und Moorgebieten und ihren reichen Jagd- und Fischgründen bildeten deutlich eine gute Grundlage für die lappische Lebensführung. Wir wissen beispielsweise, dass das Wildren hier noch bis in die 1830er Jahre lebte. ${ }^{13} \mathrm{Nach}$ Tegengren gab es um die Mitte des 18 . Jh. noch ziemlich viele Wildrene in diesen Gegenden. ${ }^{14}$ Aspelin hat nachgewiesen, dass diese relativ späten Andenken an die Lappen in dem Gebiet auf den Massnahmen beruhen können, die Karl IX. zur Entwicklung der Rentierzucht in den "'Einödgebieten von Ilmajoki" traf, die wohl - wie aus dem Briefwechsel 1607 ersichtlich - davon ausgingen, dass der Bedarf der Krone an

${ }^{9}$ Siehe T. I. Itkonen 1948 I, S. 92-97 mit Literaturhinweisen. Die Lappen, die zu dem Gut Kantala in Sääksmäki gehörten, sollen sich 'nor $i$ botne' befunden haben, was sich wohl auf die Einödgegend im Norden bis hin nach Mittelfinnland bezogen hat (T. I. Itkonen 1947 s. 48; vgl. Anmerkung 1).

${ }^{10}$ T. I. Itkonen 1948 I, S. 92; Anders Walerius, Suomi 1847 S. 48; K. B. Wiklund 1911 S. 177.

${ }^{11}$ Niilo Valonen, Vähän kyröläisistä lappalaismuistoista. Satakunta XIV. Vammala 1948 S. $85-$.

12 Viljo Alanen, Satakunnan Lappi. Satakunta IX. Vammala 1931 S. 81-.

13 "Am zähesten haben die südlichen Wildrene ihre Position in Obersatakunta behauptet, wo sich ein kleiner Stamm bis zum Jahre 1830 in den Einödgegenden von Parkano erhalten haben soll', Lauri Siivonen-Tauno Mäki, Metsäpeura, Suomen nisäkkäät 2. Helsinki 1972 S. 387.

14 Helmer Tegengren, En utdöd lappkultur i Kemi lappmark. Åbo 1952 S. 242. 
Rentieren gedeckt würde, sicher hauptsächlich durch den schwierigen Transport verursacht. ${ }^{15}$ Entsprechende Vorkehrungen wurden auch anderwärts getroffen. ${ }^{16}$ Die Lappen sollten ermuntert werden, in das Gebiet zu übersiedeln, sie sollten die Aufgabe erhalten, den Finnen die Rentierzucht beizubringen. Die Bauern in dem Bezirk durften diese Aktivitäten nicht erschweren. Die sechsjährige Steuerbefreiung, die den Ansiedlern zugesagt wurde, sowie gewisse Vorteile in Form von Saatgut sowie Alleinberechtigung zu Jagd und Fischerei zeigen, welche Bedeutung vonseiten des Staates der Ansiedlung beigemessen wurde.

Angesichts dieser Angaben fragt man sich, ob hier nicht einiges darauf hindeutet, dass es dort schon früher eine bestimmte lappische Bevölkerung gegeben hat. Massnahmen dieser Art hätten wohl kaum aktuell werden können, wenn es nicht Erfahrungen gab, auf denen man aufbauen konnte. Rentiere müssen vor allem in der intensivsten Zeit der Einödfahrten eine Voraussetzung für den Transport gewesen sein. Dies musste zu einer Zusammenarbeit geführt haben zwischen den Einödfahrern und denen, die in ihrem eigenen Kulturmilieu mit den Rentieren vertraut waren, d.h. den Lappen.

2. Ich habe in aller Kürze zeigen wollen, wie man die Verbreitung der lappischen oder auf Lappen hinweisenden Ortsnamen und die siedlungsgeschichtlichen Fakten miteinander vergleichen kann. Gründlichere Untersuchungen nach diesen Prinzipien könnten sicher zu besseren Erkenntnissen über die frühere Verbreitung der Lappen in Finnland führen. Im vorliegenden Artikel möchte ich jedoch ein begrenzteres sprachliches Material behandeln, und zwar einige lappische Lehnwörter, die vor allem in den ältesten Einödgebieten von Satakunta, Südostbottnien und Häme verbreitet sind.

Niilo Valonen hat in seinem interessanten Artikel in Satakun-

15 J. R. Aspelin, Kokoelmia muinaistutkinnon alalta. Suomi II: 9. Helsinki 1871 S. 225.

${ }^{16}$ Karl IX. ergriff Massnahmen, um die Stellung des Staates im Norden zu stärken, wodurch u.a. die Verkehrsverbindungen verbessert wurden; "die Zugrentiere waren das einzig effektive Transportmittel in diesen nördlichen Gegenden." (Israel Ruong, Samerna. Stockholm 1975 S. 96.) 
ta XIV (1948) auf einige Wörter lappischen Ursprungs in Obersatakunta hingewiesen. Bei den behandelten Wörtern, die noch 1936 den ältesten Gewährsleuten bekannt waren, handelt es sich u.a. um palas 'Hasenfährte (im Schnee oder Moos)' (Park Kih [sogar Elchfährte D. Im nördlichen Finnland hat das Wort gemäss den Sammlungen der Wörterbuchstiftung (SS) die Bedeutung 'Pfad' (Kitt Muon EnoT). In Obersatakunta hat man weiterhin das Wort kaitua 'sich entfernen, verschwinden' (Park) aufgezeichnet. Frühere Belege sind aus Ika HKyr Muon EnoT bekannt. Ein Beleg aus Jämijärvi lautet: sitte se kaituu, häipyy kuulumattomiin 'dann verschwindet es, verhallt es'. Nach dem Etymologischen Wörterbuch der finnischen Sprache (SKES) ist palas aus lapp. (lpN) balges 'Pfad' entlehnt und kaitua von gai'dât 'verschwinden, sich entfernen'. Die alternative Form pallas '(Hasen)fährte' ist belegt in Kärs Viitas PyhOl.

Terho Itkonen hat in seiner Untersuchung über die Skiterminologie auf einige finnische Wörter hingewiesen, unter denen sich offenbar eine Gruppe lappische Lehnwörter befindet, während andere Bedeutungsveränderungen zeigen, die auf lappischen Einfluss hinweisen.

Das Wort sivakka (sivakko, sipakka) 'Ski; Sumpfski; Bottichkufe' von lapp. (lpN) sâbek sâvek 'Ski' kommt ausser im nördlichen Finnland sogar im südlichen Ostbottnien und in Obersatakunta vor (S. 21), während kola 'Führungsrille am Ski', das auf lulelapp. (lpL) goalle (kầlle 'Laufrinne an der Unterseite eines Skis'; H. Grundström) zurückgehen kann, nur in Obersatakunta erwähnt wird (S. 85). Itkonen hält es nicht für unmöglich, dass kolo, eines der Synonyme, eine Volksetymologie von kola sein kann. Dann würde die Verbreitung von kola und kolo in Satakunta dem Vorkommen von sivakka entsprechen.

Sivakka erscheint auch als Element in Namen in der Nähe des Festungsbergs Siuro im oberen Teil von Kulovesi. Von hier aus konnte man bereits in der Eisenzeit den Seeweg hinauf nach Kyrösjärvi und Pyhäjärvi bewachen. ${ }^{18}$ Bei der Festung finden

1 Terho Itkonen, Suomen kielen suksisanastoa. Helsinki 1957.

18 Helmer Salmo, Satakunnan historia II. Rautakausi. Vammala 1952 S. 103. 447. 
sich die Namen Sivakannokka, Sivakanlahti und Sivakkavuori. ${ }^{19}$

Sompa (somma, somppa) in der Bedeutung 'Skistock' und nicht 'Stockteller' kann weiterhin auf lappischem Einfluss beruhen (vgl. IpN soab'be 'Stab'). Aber auch in diesem Fall ist das Verbreitungsgebiet ungefähr dasselbe.

Ich behandle in dem Zusammenhang nicht die anderen lappischen Skitermini, die in die nordfinnischen Dialekte entlehnt worden sind, sondern weise nur auf die Tatsache hin, dass sie - ähnlich wie mehrere der anderen Wörter, die ich anführen werde - ein nördliches Verbreitungsgebiet haben, das im Süden eine Spitze gegen das zentrale Finnland bildet. ${ }^{20}$ Von diesen Gebieten des Landes verfügen wir auch über ein reichhaltigeres schriftliches Archivmaterial über die lappische Besiedlung.

3. Beim Durchsehen des Finnischen Etymologischen Wörterbuches habe ich einige lappische Lehnwörter gefunden, die ausser im nördlichen Finnland vor allem im südlichen Ostbottnien und auch in Obersatakunta vorkommen. Doch haben die Wörter dort andere Bedeutungen als im Norden. Das Wörterbuch nennt die südlichen Belege nicht und es ist meine Absicht, anhand der Verbreitung einschliesslich der besiedlungsgeschichtlichen Fakten und einer Analyse der Wortbedeutungen zu erforschen, ob es sich dabei nicht auch im Süden um Lehnwörter handelt, die im Zusammenhang mit der alten finnischen Kolonisierung übernommen wurden.

Hierher gehört u.a. das Wort kurmu 'Bremse und deren Larve' (von lpN gur'bma; K. Nielsen, Larve von Oedemagena tarandi). Dieser Hautparasit ist eine ziemliche Plage der Rentiere und vermindert die Qualität der Rentierhäute bzw. -felle durch die von den Larven verursachten Löcher. ${ }^{21}$ Der Wortar-

${ }^{19}$ K. V. Kaukovalta, Pirkkalan heimo- ja keskiaika. Pirkkalan historia. Tampere 1934 S. 46. Mirja Miettinen, Vanhan Pirkkalan esihistoria. Vanhan Pirkkalan historia. Tampere 1974 S. 927.

${ }^{20}$ Über die lapp. Lehnwörter im Finn. siehe: Frans Äimä, Lappalaisia lainasanoja Suomen murteissa. JSFOu XXV, 11908.

21 Angesichts des Schadens, den diese Parasiten auf den Rentierhäuten anrichten, ist es verständlich, dass das lappische Wort ins Norwegische und Schwedische entlehnt wurde (schwed. korm, kormhål). K. B. Wiklund, Lapska namn på ren-oestriderna och deras larver. Le Monde Oriental X, 1916 S. $187-190$. 


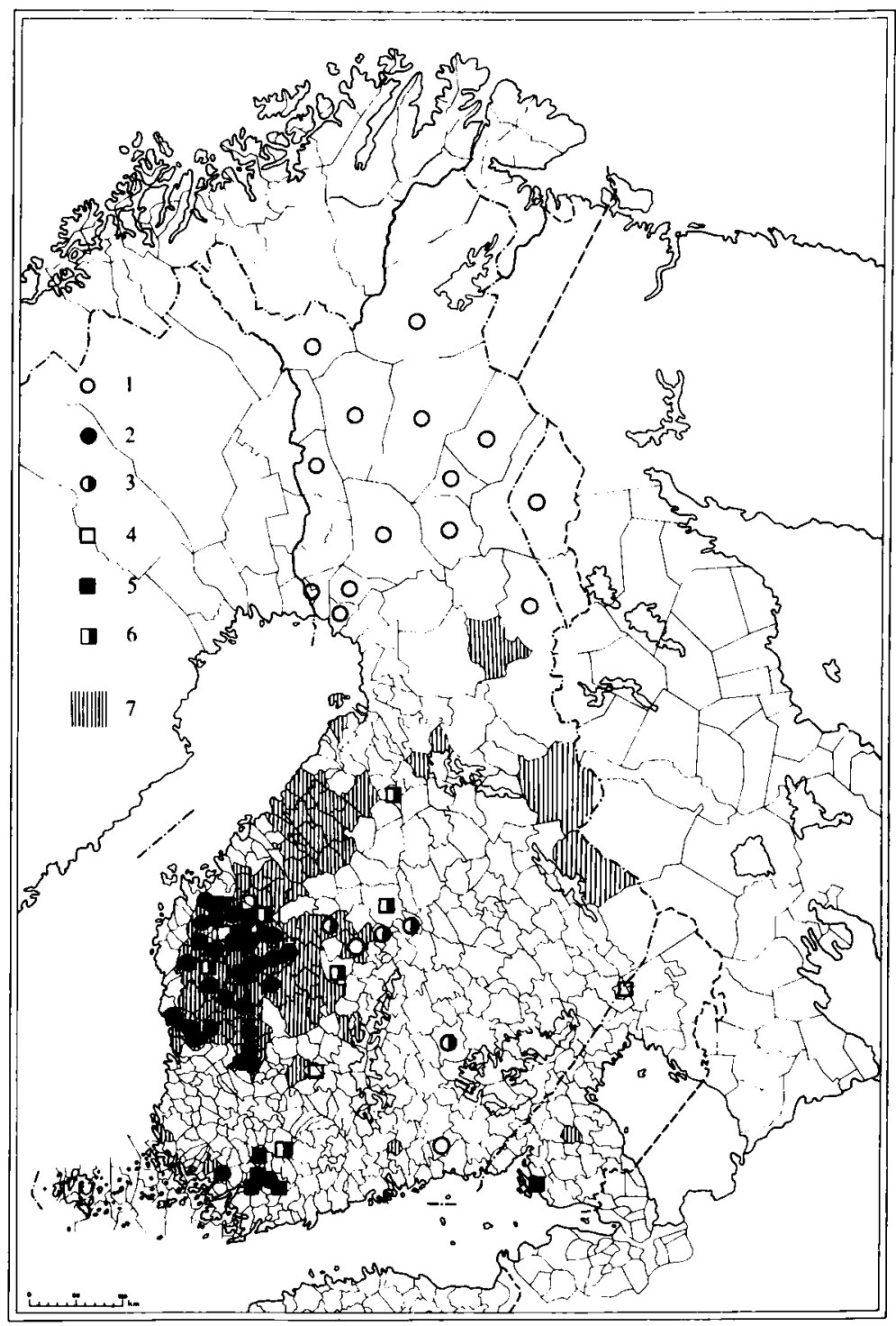

Karte 1

1 kurmu 'Bremse, Dasselfliege und deren Larve'; 'Bremsenlarve in der Rückenhaut der Kuh' (Sip); 2 kurmu 'Kuh'; 3 kurmu 'junge Ziege' (Vesa Kong); kurme id. (Kars Mik); 4 kurmu 'Sucher der besten Stücke'; 'gierig, geizig' (Vär); 5 kurmu 'dünn (Mensch)'; kurme id. (Sah.); kurmee id (Sam); 6 kurmu pejor. vom Menschen, meist von einem Jungen od. Mädchen; 7 itikka 'Kuh, Rind, Haustier'. 
tikel verschweigt, dass das Wort in Südostbottnien und Obersatakunta als Schimpfwort für Kühe bekannt ist (Laih Lapu Jur Ilm Nmo PerS Avus Töy Jal KauJ Karv Park Siikai Pom Ika HKyr Mou Suoni). Die südlichsten Belege finden sich in Paimio und Kiikala. Virtarantas Aufzeichnung ${ }^{22}$ zeigt, dass das Wort noch vor einer kurzen Zeit im Dialekt von Suodenniemi und Parkano gelebt hat.

Aus demselben Gebiet ist uns das Wort in der Bedeutung 'Sucher der besten Stücke' (Ilm Lapu) bekannt. Diese Bedeutung lässt sich wohl von den Aufzeichnungen herleiten, wo von Kühen die Rede ist, die die Zäune durchbrechen und von der frischen Saat auf dem Feld fressen (Nmo Avus Laih Pom $\mathrm{KauJ}$ ). Ausser in Sahalahti in Häme bedeutet das Wort in Muurla und Somero 'magerer Mensch'. In Kiikala gibt es die Bedeutung 'elender Mensch'. Die drei letzten Bedeutungen verdienen ihrer südlichen Verbreitung wegen beachtet zu werden. Hier im Süden finden sich sogar die Formen kurmee 'schlecht aussehender, dünner Mensch mit langem Hals' (Sam) und kurme 'dünn' (Muurl). ${ }^{23}$ In pejorativer Bedeutung wird kurmu von Mädchen verwendet in Viit Tam Lapu Nmo und von Jungen in KauJ. Ruoppila hat das Wort in pejorativer Bedeutung sogar für junge Ziegen in den Kirchspielen Kong und Vesa (S. 207) belegt; beide Orte gehören zum historischen Nordhäme.

Aus Karte 1 gehen die Belege hervor. Sie sind nach der Bedeutung und nicht nach der Form gruppiert. Ruoppila meint, die pejorative Bedeutung gehe auf die Mühe zurück, die die Ziegen ihren Besitzern machen. Als die Anzahl der Ziegen zurückging, sei das Wort auf eine lästige Kuh übergegangen. ${ }^{24}$

\footnotetext{
22 Pertti Virtaranta, Länsiyläsatakuntalaisten murteiden äännehistoria II. Vokaalit. Helsinki 1957 S. 528-529.

${ }^{23}$ Veikko Ruoppila, Kotieläinten nimitykset Suomen murteissa I. Helsinki 1943 S. 208.

24 Dagegen spricht, dass das Wort schon 1886 in Ilmajoki in der Bedeutung 'Kuh' aufgezeichnet wurde, und zwar in folgendem Zusammenhang: "Nouse, kontio, kohmikosta, Nouse, karhu, kankahasta, Ota kurmun säkehen Ja väännä se mäkehen; Eikä ikänä pidä raadon nahaat loppuman." Der Aufzeichner hat das Wort kurmu mit der Erklärung: 'lehmi', d.h. Kuh, versehen. (Suomen kansan vanhat runot XI. Etelä-Pohjanmaan runot. Helsinki 1933 S. 366.)
} 
Nach Ruoppila liegt kein Hindernis vor, gur'bma und fi. kurmu in der Bedeutung 'Bremse und deren Larve' zusammenzustellen. Es gibt ja auch noch andere Beispiele dafür, dass Insekten und Haustiere dieselbe Bezeichnung tragen (itikka, mäkärä). Dagegen ist Ruoppila der Ansicht, die südliche Verbreitung des Wortes bilde ein ernstliches Hindernis für die Annahme einer Entlehnung aus dem Lappischen.

Wenn wir aber nun - im Gegensatz zu Ruoppila - kurmu in der Bedeutung '(elende) Kuh' als Lehnwort betrachten, gibt es dann Faktoren, die für eine Kombinierung mit kurmu 'Bremse' in den nordfinnischen Dialekten sprechen? Wie kann man sich dann die Bedeutungsveränderung 'Bremse' $>$ (lästig, elend, dünn usw.) 'Kuh' ('Ziege, Mensch') erklären? Vermutlich findet sich eine Antwort, wenn man die Verbreitung des Wortes vergleicht mit dem, was wir über die finnische Besiedlungsgeschichte wissen, doch darauf kommen wir etwas später zurück.

4. Aus dem SKES erhellt auch nicht die südliche Verbreitung von kieppi (kieppä), das im Norden 'Übernachtungsgrube von Auerhuhn, Birkhuhn, Moorschneehuhn oder Hase im Schnee' bedeutet. Die Bedeutung '(durch den Wind verursachte) Höhle im Schnee; Schneewehe', die im etymologischen Wörterbuch ebenfalls fehlt, hat im Norden ungefähr dieselbe geographische Verbreitung. ${ }^{25}$ Das lappische Wort lautet (lpN) čiep'pâ, (lpL tjiehppa) 'kleine Grube im Schnee, die Waldvögel sich als Schlafplatz aufscharren' (H. Grundström). Lapp. $\check{c}$ vor einem vorderen Vokal ist durch $k$ substituiert worden. ${ }^{26}$

Aus den lexikalischen Sammlungen des SS geht hervor, dass das Wort in Obersatakunta und in Häme vor allem in der Bedeutung 'Grube (im Schnee); Schneewehe' belegt ist. Einige mehr verbreitete Belege finden sich in UusVl., KanJ (von Hämeenkylä !), Kive und Inkere. In Orivesi bedeutet das Wort 'lagernde Stelle im Getreidefeld' und in Korpilahti 'Grube im Sand'. KanP Tam Keu Tuu 'Übernachtungsplatz eines Birkhuhns (Waldvogels) im Schnee' und Hir SavT 'Hasengrube im

${ }^{25}$ Itkonen meint kieppi (lumikieppi) in einer südlichen Aufzeichnung von Kangasniemi sei ein lapp. Lehnwort. (T. I. Itkonen, Uuttu. Virittäjä 1934 S. 218.)

${ }^{26}$ Frans Äimä, JSFOu XXV, 1 S. 57. Lauri Posti, Virittäjä 1959 S. 209. 
Schnee' stehen der ursprünglichen lappischen Bedeutung nahe.

Sowohl kieppi als auch kurmu haben eine nördliche Verbreitung, die einer für lappische Lehnwörter üblichen Streuung in Nordfinnland folgt (s. die Skitermini oben). Ausserdem kommen sie - wie wir sahen - in einem kleinen Umkreis in der südwestlichsten Ecke von Häme und den angrenzenden Teilen von Varsinais-Suomi und Uusimaa vor.

Welche Funktion hatte das Wort kieppi, dass es denkbare finnische Synonyme verdrängen konnte und wie können wir die Bedeutungsveränderung 'Übernachtungsgrube (von Auerhuhn etc.) im Schnee' > '(vom Wind verursachte) Grube im Schnee; Schneewehe' erklären? Die Lösung liegt sicher in der Funktion, die das Wort früher als Jagdterminus innehatte. Die Jagd auf Waldvögel lohnte sich an solchen Stellen, wo die Tiere die Nacht zu verbringen pflegten. Man überraschte die Tiere an ihren Schlafplätzen oder blendete sie auch in der Dunkelheit durch das Licht von Fackeln. ${ }^{27}$ "Der die Natur beobachtende Einödjäger lernt bald, wo die Waldvögel, die Hasen, die Marder und anderen kleinen Tiere zu übernachten pflegen"', wie Sirelius schreibt (S. 61). In einem Beleg aus Luumäki (Taavetti) heisst es: ' Im Winter, wenn der Schnee trägt, schaut man in der Dämmerung nach dem Aufenthaltsplatz der Birkhühner. In der Nacht fing man dann die Vögel, indem man einen Kescher über die Grube legte, in der sich der Vogel befand." (SS, Kauko Oksa.)

Begreiflicherweise wussten die Lappen am besten, wo sich das Wild befand, und konnten deshalb auch den finnischen Jägern den Weg weisen. Da der Lappe der Sachverständige war, hatte seine Terminologie den Status, in den Wortvorrat des Finnen übernommen zu werden. Als die Bedeutung der Jagd später abnahm, verlor kieppi seine prägnante Bedeutung als Jagdterminus. Die Verbindung mit dem Schnee blieb erhalten.

Es gibt noch andere Beispiele dafür, dass lappische Schneetermini ins Finnische entlehnt wurden. Von ihnen soll hier kiekerö, kiekero, kiekirä von lapp. čiegâr erwähnt werden. ${ }^{28}$

${ }^{27}$ U. T. Sirelius, Suomen kansanomaista kulttuuria I. Helsinki 1919 S. 64-65 (Abb. 2).

${ }^{28}$ Frans Äimä, JSFOu XXV, 1 S. 20. 
Dieses Wort war nämlich ursprünglich sicher ein Jagdterminus. Heute bedeutet das lappische Wort 'von Rentieren im Winter aufgescharrtes und abgeweidetes Gebiet' (H. Grundström). Eine exaktere Bedeutung wäre 'schmales von Rentieren niedergetretenes Schneestück am Rand von Weidegebiet'. In der Beschreibung der Jagd auf Wildrene im 17. Jh. schildert Johannes Tornaeus, dass man die Rentiere in die äusseren Teile des Weidegebietes scheuchte, wo man sie mit Pfeilen oder Speeren tötete oder in Schlingen fing. Als Terminus für diese Jagd verwendet Tornaeus das Wort kiekero. ${ }^{29}$

5. Das Gemeinsame an der Verbreitung von kurmu und kieppi ist, dass sie innerhalb von Obersatakunta eine schmaler werdende Spitze nach Süden bilden. Die Verbreitung der Wörter ist fast identisch in dem Gebiet und beschränkt sich auf den Umkreis des alten Grosssprengels Kyrö, dessen frühere Einödkultur wohlbekannt ist. ${ }^{30}$ Der See Kyrösjärvi mit seinen in verschiedene Richtungen gehenden Wasseradern wurde zum Zentrum für das ganze Kirchspiel, wo sich die Bevölkerung verdichtete. Die ersten Spuren menschlicher Besiedlung haben wir bereits aus der Eisenzeit. Man nimmt an, dass die Einwanderung aus dem Tal des Kokemäenjoki kam.

In der Zeit, da die Wasserwege die wichtigsten Verkehrslinien bildeten, wurde die Verbreitung der Bevölkerung durch die Höhenrücken Hämeenkangas und Pohjankangas begrenzt. Sie bildeten nämlich die Wasserscheide zwischen den alten Grosssprengeln Sastamala und Kyrö (Hämeenkyrö) und damit auch eine natürliche Grenze zwischen ihnen. Gleichzeitig boten die Bergrücken eine Möglichkeit für den Verkehr in Nord-SüdRichtung vor allem für die Einwohner von Hämeenkyrö. ${ }^{31} \mathrm{Da}$ die Landrücken in der Gegend von Kankaanpää am niedrigsten sind, erfolgt die Expansion der Einwohner in westlicher Richtung. Dies geschah nach Jaakkola gerade zu der Zeit, als die Einödfahrten nach Norden ins südliche Ostbottnien durch die

\footnotetext{
29 I. Ruong 1975 S. 19.

${ }^{30}$ Seppo Suvanto, Satakunnan historia III. Keskiaika. 1973 S. 208-212, $250-267$.
}

31 Armas Luukko 1950 S. 9-. 
zunehmende feste Besiedlung am Unterlauf des Kyröjoki verhindert wurden. ${ }^{32}$

Beachtenswert ist, dass HKyr Vilj Ika Jämi Park Kih, d.h. die zentralen Teile des alten Kirchspiels Kyrö, sprachlich eng zusammengehören mit KanP Karv und HonJ auf der westlichen Seite der Bergrücken. ${ }^{33}$ Wenn es sich so verhält, dass die typischen Lautwechsel $\bar{a}>\bar{a}>\bar{o}$ und $\bar{a}>\bar{\varepsilon}>\bar{e}$, die wir in HKyr Vilj Ika Jämi KanP Park wiederfinden ${ }^{34}$, ursprünglich sind - wie Virtaranta behauptet - und nicht durch Einwirkung der Savo-Dialekte entstanden sind, so haben wir hier einen Hinweis, wo das alte Kerngebiet von Kyrö zu suchen ist. Virtaranta erwähnt (S. 71), dieser Lautwechsel sei sporadisch sogar in Pom und Siikai vorgekommen, was als weiteres Anzeichen für die westliche Richtung der Bevölkerungsbewegung betrachtet werden kann.

Die wichtigste Wasserstrasse verlief bereits frühzeitig vom Kyrösjärvi nach Norden via Parkanojärvi zum Kyröjoki ${ }^{35}$, und ein Winterweg folgte noch in später Zeit dem Schiffsweg, was auf die Bedeutung dieser Gegend für den Verkehr hinweist. ${ }^{36}$ Alle die genannten Wege laufen in Hämeenkyrö zusammen und natürlich gab es von dort Verbindungen nach dem Süden, nach Rautavesi und Kulovesi. Die Verkehrsrichtung war auch durch die kirchliche Gebietseinteilung verstärkt worden. Wir wissen, dass die Kirche in Karkku lange Zeit die einzige war und dass man auch von weit aus dem Norden hierher zu fahren hatte, bis die erste Kirche in Viljakkala errichtet wurde. Über diese Fahrten nach Karkku berichtet die Überlieferung in den Grenzgebieten von Südostbottnien und Obersatakunta noch heute. ${ }^{37}$

32 Jalmari Jaakkola, Pohjois-Satakunnan vanha eräkulttuuri. Satakunta V. Porvoo 1925 S. 27-28. Vgl. zur Einödkultur in Kyrö: ders., Pirkkalaisliikkeen synty. Turku 1923.

33 Perti Virtaranta 1946 S. 6.

${ }^{34}$ Pertti Virtaranta, Ikaalisten seudun murteen savolaisuudet. Satakunta ja satakuntalaisia II. Pori 1951 S. 71.

${ }^{35}$ Armas Luukko 1950 S. 5.

36 Viljo Alanen, Kyrönkankaan vanha talvitie. Satakunta XIII. Vammala 1946.

${ }^{37}$ Armas Luukko 1950 S. 10. J. R. Aspelin, Kokoelmia muinaistutkinnon alalta. Suomi II: 9. Helsinki 1871 S. 162. 
Wir können somit feststellen, dass das Erwerbsleben, die Verkehrsverhältnisse und die kirchlichen Verhältnisse die Menschen innerhalb des Kirchspiels vereinten. Als ein Resultat dieser soziologischen Faktoren entstand eine sprachliche Einheitlichkeit. Es ist daher verständlich, dass man mit Jagd und Fischerei verbundene Termini findet, die nur hier belegt sind. Ein solches Wort lautet pätäs 'Brustfleisch des Vogels' ${ }^{38}$, das seiner Verbreitung nach mit kurmu verglichen werden kann, während dagegen rökäs 'Kaulbarsch' in seinem südlichen Gebiet eine Übereinstimmung aufweist mit kieppi. Die Belege um Längelmävesi sind besonders aufschlussreich (rökäs ausser in VesL auch in KanA Sah Erä Län; kieppi ausser in Mes auch in Oriv KuhL Pad Sys Jäms KuoV KosP KorL). Wie kann man da erklären, dass ein Wort mit Anschluss in Obersatakunta im heutigen Häme auftritt? Es ist einfach so, dass die beschriebene Gegend einmal zu Obersatakunta gehörte.

Sowohl die Archäologie als auch die Geschichtsforschung vermitteln uns Kenntnisse über die Siedlungsbewegungen. Wir wissen aus den archäologischen Funden, dass sich die Besiedlung in östlicher Richtung ausdehnte und in vorhistorischer Zeit zuerst das heutige Messukylä, Lempäälä, Vesilahti und Kangasala erreichte ${ }^{39}$, während die Ufer der Seen Pyhäjärvi und Näsijärvi unbesiedelt blieben. ${ }^{40}$ In diesem Zusammenhang verdient die Tatsache Erwähnung, dass rökäs und kieppi in der Umgebung des Näsijärvi fehlen. Die feste Besiedlung erreichte auch Vanajavesi, doch geschah das in der Weise, dass der Kontakt zu den ursprünglichen Gegenden abriss. ${ }^{41}$ Die Landstriche um den Pyhäjärvi, besoriders die südlichen Ufer, wurden erstaunlich spät besiedelt. All dies scheint dazu beigetragen zu haben, dass die Grenze zwischen Satakunta und Häme von einem frühen Zeitpunkt an in diesen Gegenden verläuft. ${ }^{42}$

Von Längelmävesi aus verbreitete sich die Besiedlung weiter

${ }^{38}$ Niilo Valonen, Yläsatakuntalaisia sanoja anastavien elinkeinojen alalta. Satakunta XIII. Vammala 1946 S. 176- 178.

${ }^{39}$ Helmer Salmo 1952 S. 137, 141. Seppo Suvanto 1973 S. 218.

40 Juhani Saarenheimo, Vanhan Pirkkalan historia. Tampere 1974 S. 35.

41 Jalmari Jaakkola 1956 S. 277.

42 Seppo Suvanto 1973 S. 217. 
in nordöstlicher Richtung ${ }^{43}$, während sie von Vanajavesi eine südöstliche Richtung nahm und erst später die mittleren und nördlichen Ufer des Päijänne berührte. ${ }^{44}$

Die besondere Verbreitung des Wortes spiegelt somit die alten Einödfahrten samt der nachfolgenden Besiedlung wieder. Sowohl der frühe Charakter der Besiedlung als auch deren Kontinuität sind von Bedeutung für die Überlieferung des Wortes bis in unsere Tage. Diese Faktoren können beim Näsijärvi mitgespielt haben, der lange Zeit eine Art Allmende bzw. Jedermannsland war, d.h. es wurden Einödfahrten dorthin unternommen, doch die feste Besiedlung kam relativ spät.

Mehrere Einzelheiten sprechen dafür, dass kieppi während der ersten Einödzeit übernommen wurde: a. die Gebiete um den Längelmävesi gehören nach der Verbreitung des Wortes zu urteilen noch zu Obersatakunta, b. um den Näsijärvi fehlen Belege, was ebenfalls auf frühere Verhältnisse hinweist und c. der von Häme ausgehende Einfluss aus der Richtung des Vanajavesi scheint noch nicht begonnen zu haben.

6. Nun können wir zurückkehren zu kurmu, verwendet als Schimpfwort für Kühe. Wir hatten bereits zwei andere gemeinsame Bezeichnungen für Insekten und Rinder genannt (itikka, mäkärä). Itikka in der Bedeutung 'Kuh, Rind, Haustier' fällt sprachgeographisch gut zusammen mit kurmu 'Kuh' (Karte 1). Ruoppila (1943 S. 135) hält das Wort itikka für ein Diminutivum von jenem Wortstamm, der z.B. auftritt in itää 'keimen', itu 'Keim', itiö 'Spore'. Die Verhältnisse im Pflanzen- und Tierleben sind nach Ruoppila derart analog, dass es nicht merkwürdig ist, dass das Wort als Bezeichnung für Insekten oder sehr kleine Tiere überhaupt begegnet. Die Anwendung des Wortes für Rinder kann seines Erachtens möglicherweise ein Euphemismus sein. Die Entwicklung kurmu 'Bremse' > kurmu 'Kuh' könnte aufgrund dessen erklärt werden, dass man sich der Beziehung itikka 'Mücke' - itikka 'Kuh' in dem gleichen

${ }^{43}$ Die westlichsten Dörfer von Kangasala hatten in der Gegend von Kuorevesi Einödgebiete (Seppo Suvanto 1973 S. 96); vgl. Vilho Niitemaa, Hämeen keskiaika. Hämeen historia I. Hämeenlinna 1955 S. 278.

44 Jalmari Jaakkola 1956 S. 278. 
Gebiet wohlbewusst war. Als Bezeichnung für ein Insekt ist das Wort im Finnischen allgemein. Kurmu in seiner ursprünglichen Bedeutung begegnet im südlichen Finnland lediglich in dem Beleg aus Sippola und Karstula 'Bremsenlarve in der Rückenhaut der Kuh'. Das Auftreten des Wortes in Südostbottnien und Obersatakunta lediglich unterstützt die Theorie einer Entlehnung aus dem Lappischen. Wir haben ja feststellen können, dass es eine Mehrzahl sicherer Lehnwörter in der Gegend gibt.

Bei kurmu ist die Verbreitung besonders auffallend, denn das Wort deckt ganz und gar jenes Gebiet, das wir als weitesten Interessenbereich von Kyrö nachgewiesen haben. Im südlichen Ostbottnien finden wir das Wort ebenfalls im Tal des Kyröjoki von den Quellflüssen bis zur Mündung. Von hier gingen die Fahrten der Bewohner von Satakunta nach Norden. Einige Belege scheinen auch die Expansion auf die Westseite von Hämeenkangas und Pohjankangas widerzuspiegeln, die oben erwähnt wurde (Siikai Pom MerK).

Welche Funktion hat das Wort nun gehabt, wodurch erhielt es den Status, dass es in den Dialekt der Umgebung aufgenommen wurde? Der Grund war sicher derselbe, der zur Übernahme ins Norwegische und Schwedische führte - der Umgang mit Pelzwerk und Tierhäuten und die Beurteilung von deren Qualität. Der Handel mit Tierhäuten und Pelzwerk und die Jagd auf Pelztiere war ja ein Teil der Einödwirtschaft. Das Besteuerungssystem, das sog. Kyrö-Recht, das auf dem Ertrag der Pelztierjagd basierte, zeugt von der Bedeutung, die dieser Erwerbszweig hatte. Da die Lappen mit der grössten Wahrscheinlichkeit auch im Innern Finnlands an ihre finnischen Besteuerer Pelzwerk und Tierhäute abgaben, findet die Entlehnung des Wortes eine natürliche Erklärung. Das Fehlen des Wortes in den zentralen Teilen von Häme kann darauf beruhen, dass die entsprechenden Kontakte mit den Lappen nicht so intensiv oder anhaltend waren oder auch nicht so lange währten wie in den oberen Teilen von Satakunta. ${ }^{45}$

Ausserdem war es aber besonders ein Umstand, der dazu

45 Väinö Voionmaa, Suomalaisia keskiajan tutkimuksia. Veroja, laitoksia, virkamiehiä. Porvoo 1912 S. 58-59. 
beitrug, dass der Bedeutungswechsel 'Bremse' > 'Kuh' möglich wurde. Die Kühe hatten nämlich in alter Zeit ähnliche Hautparasiten wie die Rentiere heute. In einer Aufzeichnung heisst es: "Früher waren die Dasselbeulen gewöhnliche Parasiten der Kühe. Am Körper der Kuh bildeten sich geschwürartige Beulen mit einem Loch an der Spitze. Wenn man an der Beule drückte, sprang eine dicke Larve heraus. Die Dasselbeulen führte man darauf zurück, dass die Kuh im Sommer von der Dasselfliege gestochen wird." (SS, Piippola, Eeva Aulis 1939.)

Ein anderer Informant beschreibt, welche Plage der Parasit verursacht: "Die Dasselfliegenbeule ist auf dem Rücken der Kuh, nicht an den Seiten. Sie durchstösst die Haut. Die Larve kommt heraus und es eitert. Die Kühe auf diesem Hof hatten so schrecklich viel davon." (SS, Valtimo, Anja Hurme 1952.)

In einem Milieu, das sowohl aus einer entwickelten Einödkultur als auch aus einer zunehmenden Viehzucht bestand, wurde kurmu zu einem Ausdruck mit spezieller Attraktionskraft, d.h. neben der ursprünglichen Bedeutung konnten leicht andere auftauchen. Das Wort konnte zwar nicht das übliche finnische permo, permu, permonen 'Dasselfliegenbeule; i.a. Hautparasit (Hypoderma bovis)' verdrängen, sondern wurde ein Schimpfwort für eine unruhige, lästige, magere Kuh, die - in einer früheren Periode der Verwendung des Wortes - von Hautparasiten geplagt war.

Vor dem Hintergrund ist auch der Bedeutungsübergang 'jämmerliche, elende, magere Kuh' > 'elender, magerer Mensch' verständlich. Wenn wir weiterhin die Verben kurmuttaa, kurmottaa 'bedrängen, bestrafen, quälen' als denominale Ableitungen von kurmu betrachten, würde die Bedeutung des Wortes in der Plage liegen, die das Tier durch die Parasiten erleidet. ${ }^{46} \mathrm{Im}$ Lappischen bedeutet die entsprechende kausative Ableitung gur'modit (lpL kur'mōtit; H. Grundström) 'durch Erzeugung von Dassellarven eine Haut löcherig machen (von der Hautbremse des Rentiers, Oedemagena tarandi)' ${ }^{47}$

${ }^{46} \mathrm{Zu}$ dem Ableitungstyp s. Lauri Hakulinen, Suomen kielen rakenne ja kehitys. Keuruu 1963 S. 238.

47 Harald Grundström, Lulelapsk Ordbok, Lulelappisches Wörterbuch. Lund-Uppsala 1946-1954. 
Das Verb ist weiter verbreitet als das Substantiv, findet sich jedoch im gleichen Umkreis. Die Wörterbuchangaben (Ganander, Lönnrot) ${ }^{48}$ können darauf hinweisen, dass das Substantiv früher bekannter gewesen ist.

7. Wir haben uns bisher mit dem Vorkommen der beiden Wörter in Satakunta beschäftigt. Kieppi existiert gleichwohl weiter nach Osten hin. Wie wir sehen werden (Karte 2), spiegelt die Verbreitung auch dort die alten Kontakte zwischen der finnischen und der lappischen Bevölkerung wider. Wenn wir von den bekannten siedlungsgeschichtlichen Fakten ausgehen, kann man wohl eine nördliche Region in Häme absondern, die durch einen frühen Einödverkehr gekennzeichnet ist und jenen Teil von Häme ausmachte, wo die finnisch-lappischen Kontakte intensiv genug waren, um sprachliche Andenken im Finnischen zu hinterlassen.

Bereits im 7. Jh. kann eine Besiedlung in Sysmä49 und etwas später in Heinola (S. 53) festgestellt werden. Padasjoki, Kuhmoinen, Sysmä und Heinola erhalten gegen Ende des ersten Jahrtausend eine immer dichtere Besiedlung. ${ }^{50}$ Wenn die Besiedlung auch sprungweise vor sich ging, kann - den archäologischen Funden nach zu urteilen - ein Fahrweg von u.a. Längelmävesi über Sysmä ausgemacht werden. Seine Fortsetzung führte teils nach Joutsa (S. 225), teils nach Heinola. ${ }^{51}$ Jaakkola hat darauf hingewiesen, dass die Einödfahrten von Häme in ihrer frühesten Periode erstaunlich rasch in östliche Richtung gingen. Die gutbekannte nördliche Fahrtrichtung ist eine spätere Erscheinung. Aus der Wikingerzeit haben wir Gräber in Hartola und Pertunmaa. ${ }^{52}$ Zwei Grabfunde in Mikkeli und Einzelfunde aus Pertunmaa, Mäntyharju (vgl. Jaakkola 1956: 211) und Savitaipale, die aus vorhistorischer Zeit stammen, weisen eindeutig westliche Züge auf. ${ }^{53}$

\footnotetext{
48 Vgl. Veikko Ruoppila 1943 S. 208.

${ }^{49}$ Ella Kivikoski 1955 S. 44.

so Vilho Niitemaa 1955 S. 235.

si Jalmari Jaakkola 1956 S. 211

52 Ella Kivikoski 1955 S. 54.

${ }^{53}$ Eero Kiviniemi, Suomen partisiippinimistöä. Helsinki 1971 S. 73.
} 


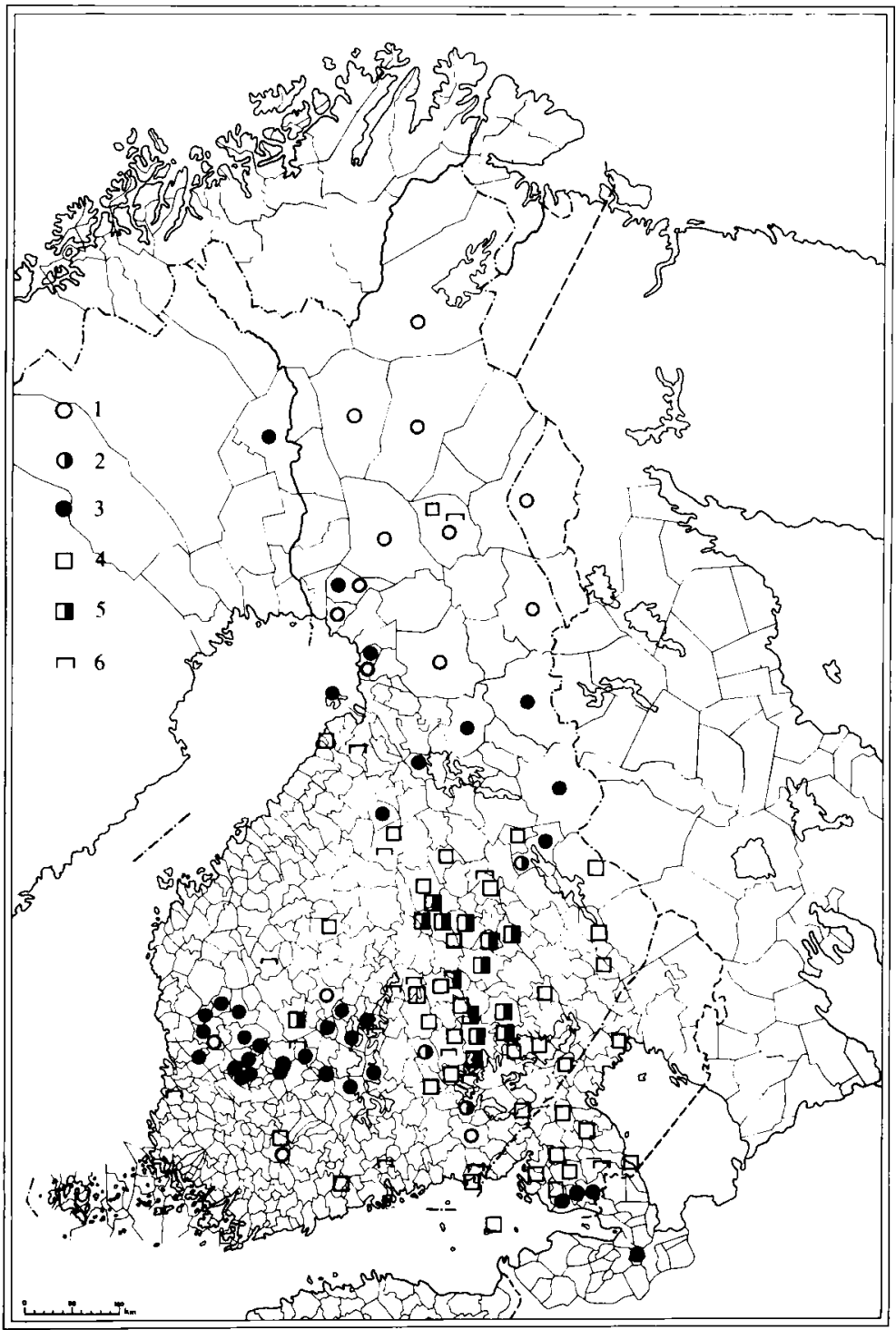

Karte 2

1 kieppi '(Übernachtungs)loch vom Birkhuhn (Waldvogel) im Schnee'; kieppä id. (Tervola Ii Sall Sod Kitt Luu); kieppa id. (KemJ); 2 kieppi 'Hasenloch im Schnee'; 3 kieppi '(vom Wind verursachte) Grube im Schnee; Schneewehe'; kieppä id. (Tervola Ii); kieppa id. (Kärs); 4 kieppi '(Schnur-, Seil-, Birkenrinden-)knäuel; (Schlangen)ring; Knoten'; 5 kieppu = kieppi (4); 6 kieppi in übertragener Bedeutung (z.B. 'schlechte Gesellschaft'). Im Text kieppi ${ }^{1}=3$ und kieppi $i^{2}=4$ und 5 . 
In diesem Zusammenhang kann es auch aufschlussreich sein, die lange und deutliche Grenze zwischen dem Gebiet Asikkala und Sysmä $\mathrm{zu}$ beobachten. ${ }^{54}$ Sie verlief von den Gebieten südlich von Padasjoki nach Mäntyharju. Die Grenze wurde i.J. 1443 festgelegt (S. 346), ging aber begreiflicherweise auf eine viel ältere Zeit zurück. Unsere Belege für kieppi finden sich nördlich dieser Grenze.

Eine andere Einöd- und Besiedlungslinie in Häme tritt noch deutlicher hervor. Sie verläuft über Sääksmäki (aus den Gebieten um Vanajavesi) über Hauho, Tuulos, Lammi und Hollola weiter nach Osten. In seiner Untersuchung der finnischen Partizipiennamen findet Kiviniemi, dass die Namen mit Tyrise$v \ddot{a}-$, Helisevä- und Koriseva- ${ }^{55}$ (Karten S. 66, 103) deutlich der östlichen Verbreitung der Besiedlung in der Eisenzeit folgen. Diese Namen finden sich in reicher Anzahl auch am Nordwestufer des Ladogasees. In Karelien haben die Namen eine Verbreitung, die fast zusammenfällt mit jenen Plätzen, wo man alte Gräber eindeutig westfinnischen Charakters gefunden hat. Das älteste Grab in Sakkola stammt ca. aus dem 9. Jh. (S. 75).

Im Hinblick darauf, dass die genannten Ortsnamentypen eng verankert sind in der frühen Besiedlungsexpansion von Häme, kann ein Vergleich mit kieppi aufschlussreich sein. Falls unsere Annahme stimmt, dass kieppi eine Entlehnung aus dem Lappischen ist, zeigt seine Verbreitung, wo sich der Grenzbereich zwischen finnischer und lappischer Besiedlung zu jenem frühen Zeitpunkt befand. In den etwas südlicheren dichter besiedelten Bezirken von Satakunta und Häme ist das Wort nicht eingedrungen. Die Belege in KanP Keu Luu Tam 'Birkhuhn-Grube im Schnee' und Hir SavT 'Hasengrube im Schnee' können als Reliktbedeutungen angesehen werden, die den von uns angenommenen Bedeutungswechsel unterstützen, der das Resultat 'Grube, Höhle im Schnee; Schneewehe' hatte.

Das Vorkommen des Wortes in der angeführten Bedeutung auf der Karelischen Landenge ist schwerer zu deuten (Uus Vl KanJ Kive und Inkere). Die einfachste Erklärung wäre, dass

\footnotetext{
54 Vilho Niitemaa 1955 S. 377.

ss Eero Kiviniemi 1971.
} 
sich hier Spuren einer Häme-Besiedlung zeigen. Die Partizipnamen besitzen ja eine regelmässige Verbreitung in diesem Gebiet (Kiviniemi 1971: 114). Das Vorkommen von HämeNamen ist von Jouko Vahtola ${ }^{56}$ gerade für UusVl KanJ und Kive festgestellt worden. Auch der charakteristische Jagdterminus aus Häme, virka, hat drei Belege in der Umgebung (S. 173).

Eine andere Erklärung könnte sein, dass das Wort in den bislang genannten Bedeutungen im Osten verdrängt oder überlagert wurde durch kieppi ${ }^{2}$ 'Knäuel, Rolle, Ring (aus Garn, Strick); Ring (den die Schlange bildet); Haarknoten, Zopf . Wie die Karte zeigt, hat das Wort eine östliche Verbreitung. So kann kieppi ${ }^{1}$ 'Grube im Schnee; Wehe' auf der Karelischen Landenge (Uus Vl KanJ Kive und Inkere) zu einem früheren Zeitpunkt eine direkte Verbindung zur "Frontlinie" von Häme gehabt haben. Die dialektgeographischen Verhältnisse unterstützen eine solche Verbindung ebenfalls. ${ }^{57}$

Hinter dem Bild, das die Verbreitung von kieppi ${ }^{1}$ 'Schneegrube; Wehe' resp. kieppi ${ }^{2} \sim$ kieppu 'Rolle, Ring; Haarknoten usw.' bietet, kann man die Folge der westlichen und östlichen Dialektkonfrontation verspüren. Wenn wir annehmen, dass kieppi sich ungefähr auf dieselbe Weise in östlicher Richtung verbreitet hat wie die Partizipnamen, würden wir ein zusammenhängendes Gebiet bis zu den Gegenden nordwestlich des Ladoga und bis zur Karelischen Landenge erhalten.

Bei dem kulturellen und sprachlichen Einfluss, der sich später durch die Karelier und Savoer aus dem Osten geltend macht, scheint kieppi seine Bedeutung als Jagdterminus eingebüsst zu haben, wodurch der Übergang zu kieppi ${ }^{2}$ (statt kieppi ${ }^{1}$ ) zustande kam. Die Zeit für diese ostfinnische Expansion in westlicher Richtung kann in grossen Zügen bestimmt werden mit Hilfe jenes Zeitpunkts, zu dem die archäologischen Funde einen veränderten Charakter aufweisen. Die westlichen Merkmale der Funde am Ladogasee hören im 12. Jh. auf. ${ }^{58}$

\footnotetext{
56 Jouko Vahtola, Paikannimistö Tornionlaakson asutushistorian lähteenä. Oulu 1975. Typoskript, aufbewahrt im Historischen Institut der Universität Oulu.

${ }^{57}$ Lauri Kettunen, Suomen murteet II. Helsinki 1930 S. 169-176.

${ }^{58}$ Ella Kivikoski, 1955 S. 73.
} 


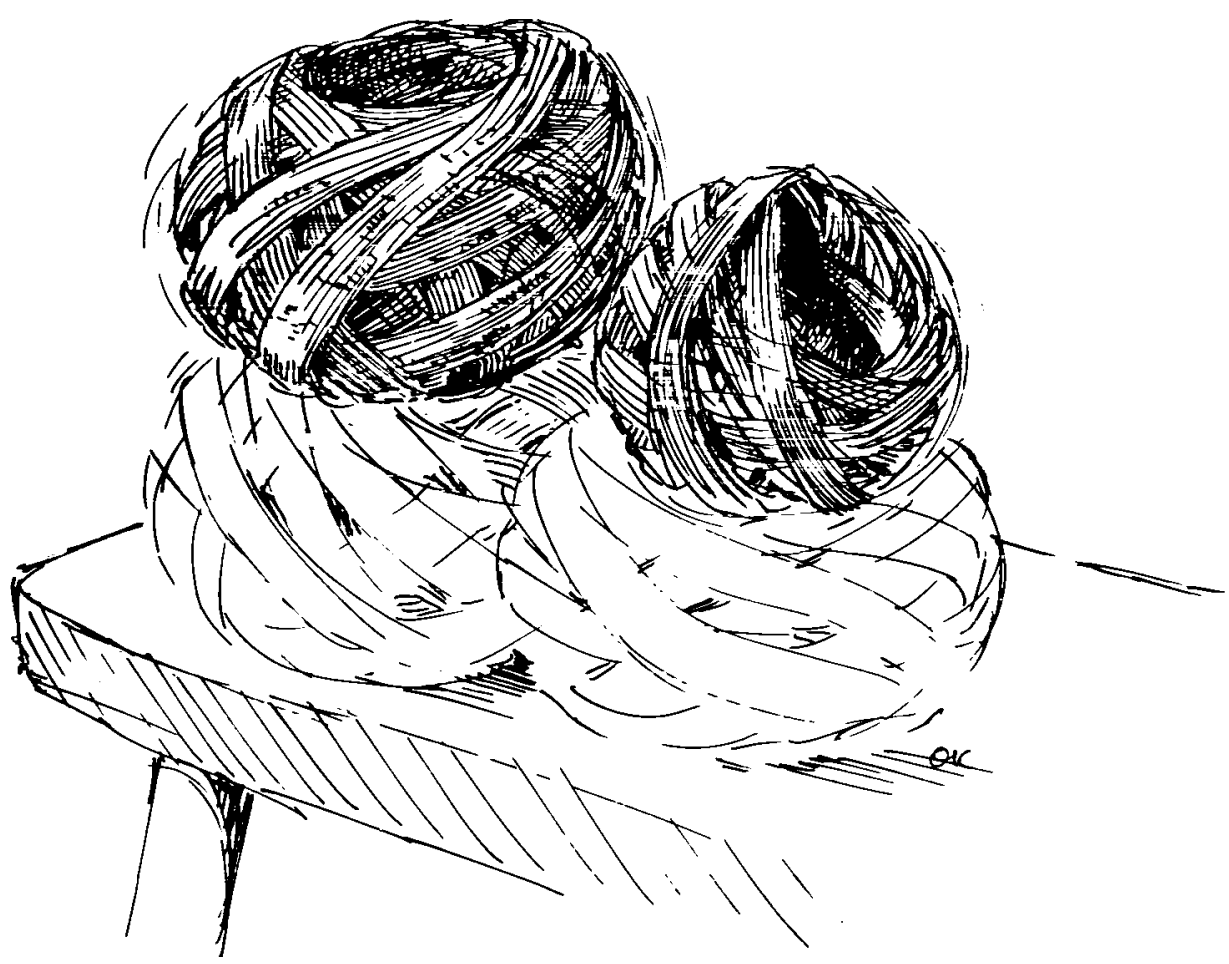

Tuohikieppi, nach einer Abbildung in Valonen 1952 S. 18.

Die Frage nach der Ursache der zwei verschiedenen Bedeutungen im Westen und Osten bleibt bestehen, ob sich das Wort nun - ähnlich wie die Törisevä-Namen ${ }^{59}$ - mit der frühen karelischen Expansion ausgebreitet hat oder ob es direkt aus dem Lappischen entlehnt wurde. Im letzteren Fall liegt hier eine gute Parallele vor in fi. pönkä ponka, wohl von lapp. (lpL) buog'ge (puoggē, H. Grundström). ${ }^{60}$ Hinsichtlich der Verbreitung unterscheidet sich kieppi ${ }^{2}$ nicht nennenswert von diesem Wort.

8. Kleine Gruppen von Kareliern müssen Einödfahrten schon vor jener Zeit unternommen haben, wo die archäologi-

s9 Eero Kiviniemi 1971 S. 77-.

${ }^{60}$ Eero Kiviniemi, Pönk(k)ä-nimet. Kalevalaseuran vuosikirja. Helsinki 1978, Karten S. 32-33. 
schen Funde einen östlichen Einfluss aufweisen. Die Funde berichten ja von einer festen Besiedlung. Es liegt auf der Hand, als einen der Wege jene Wasserstrasse anzunehmen, die den Ladogasee mit dem Fluss Pyhäjoki verband, der in den Bottnischen Meerbusen mündet. Kustaa Vilkuna hat jene Strasse beschrieben, die die karelische Besteuerung im Norden ermöglichte und die in hohem Grade die Grenzziehung im Frieden zu Schlüsselburg bestimmen sollte. ${ }^{61}$ Die Besteuerung der Lappen und der Handel mit ihnen vollzog sich eindeutig an diesem Fahrtweg. Vielleicht passte sich gerade die lappische Bevölkerung selbst dem Hauptweg der Handelsleute an, in derselben Weise wie es innerhalb der Gegend von Lule-Lappmarken in Schweden geschah, wo die Lappen ihre Winterdörfer an geeigneten Stellen in der Nähe des Fahrtweges der Handels- und Besteuerungsleute errichteten. ${ }^{62}$ Vilkuna hat jedenfalls unter Hinweis auf Ortsnamen, die teils das lappische Wort für Dorf enthalten, (sii'dâ 'Dorf'; Siilin-, Siitin- [in der Akte von Schlüsselburg Sithi] < (lpN) sii'dâ [lpL sii'ta 'Lappenlager', d.h. Vereinigung von Lappenfamilien, die ihre Zelte an derselben Stelle haben, H. Grundström], teils den Platz für das Winterdorf anzeigen (Talvisalo etc.), die Möglichkeit angedeutet, dass es in Siilinjärvi, Joroinen, Sääminki, Jääski und Keitele zentrale Plätze für die Bevölkerung der Lappendörfer gab. ${ }^{63}$ Diese Plätze waren begreiflicherweise Zentren für den Warentausch und die Besteuerung. Nicht nur die Ausbeute von Pelztierjagd und Vogelfang war in diesem Zusammenhang wichtig; Produkte des Rentiers waren als Waren sicher auch geschätzt.

Hatte das Wort (čiep'pâ $>$ ) kieppi hier nicht länger eine Funktion als Jagdterminus, ähnlich wie in einer früheren Periode in Satakunta und Häme? In seiner ursprünglichen Bedeutung war das Wort ja von jenen einzelnen Einödfahrern verwendet worden, die schon frühzeitig aus Häme kamen. Der

${ }^{61}$ Kustaa Vilkuna, Pähkinäsaaren rauhan raja kansatieteellisessä katsannossa. Historiallinen aikakauskirja. Helsinki 1960 S. 407-.

${ }^{62}$ Filip Hultblad, Övergång från nomadism till agrar bosättning i Jokkmokks socken. Lund 1968 S. 76.

${ }^{63}$ Kustaa Vilkuna, Mikä oli lapinkylä ja sen funktio? Kalevalaseuran vuosikirja 51. Helsinki 1971. 
Ortsname Siilin- enthält ja das Merkmal der Häme-Vertretung $(-l-)$ des Dentalspiranten. ${ }^{64}$

Kieppi $(<$ čiep'pâ) hat dagegen hier ein Synonym im Wort uuttu $\sim u u$, auch dies ist wahrscheinlich eine lappische Entlehnung. ${ }^{65}$ Das Wort ist nördlich der Nordgrenze von kieppi ${ }^{1}$ verbreitet. Uuttu bezeichnet auch einen Nistkasten aus einem ausgehöhlten Baumstamm, den man draussen anbrachte und zu bestimmten Zeiten im Jahr nach Vogeleiern untersuchte. ${ }^{66}$ Neben der primitiven Fangmethode, dass man die Waldvögel an ihren Übernachtungsplätzen fing, gab es weiter im Norden auch den Brauch, Nistkästen auszusetzen. Dies kann hinweisen auf milieumässige oder innerhalb der Lappenbevölkerung variierende kulturelle Verhältnisse. Die Tatsache selbst kann die Möglichkeiten von kieppi beeinflusst haben, als Ausdruck in Verbindung mit der Jagd bestehen zu bleiben.

9. Es gibt aber auch noch andere Verhältnisse, als die eben angeführten, die die Entwicklung beeinflusst haben können, so dass die Bedeutung von kieppi $(<$ čiep'pâ) nicht der in Satakunta und Häme folgte (zu kieppi ${ }^{1}$ ) sondern ihren eigenen Weg ging (zu kieppi ${ }^{2}$ ). Ich denke an kulturelle Einflüsse von aussen.

$\mathrm{Zu}$ den vielen verschiedenen Faktoren, die die Verhältnisse innerhalb einer Kultursphäre verändern, gehört nicht nur eine innere Entwicklung, sondern auch ein Einfluss von aussen z.B. in Form der Nachfrage nach Dienstleistungen und Waren. Wir können sagen, dass die Dienste der Lappen im Zusammenhang

${ }^{64}$ Kustaa Vilkuna 1971 S. 221. Vgl. Pertti Virtaranta, Pääpainollisen tavun jälkeisen soinnillisen dentaalispirantin edustus Suomen murteissa. Helsinki 1958.

${ }^{65}$ Siehe K. B. Wiklund, Ausnahmen. MSFOu 67 S. 402-, wo der unerwartete Vokal in der zweiten Silbe eines lapp. Lehnwortes $(-u)$ erklärt wird: " Das "dunkle" $-\hat{a}$ oder das noch ältere $-\alpha$ haben die finnen dann in der stellung nach $-v \nu$ - (oder nach $u$ - eher $-u$ - $u$-) als $-u$ aufgefasst und wiedergegeben, was dann endlich das jetzige $u u$ gegeben hat" (S. 414). T. I. Itkonen schliesst sich der Etymologie an (T. I. Itkonen, Uuttu. Virittäjä 1934 S. 216.), während SKES dagegen eher vermutet, dass $u u$ das Grundwort ausmacht für die Ableitungen uutto, uuttu etc.

${ }^{66}$ Eine detaillierte Beschreibung des Sammelns von Eiern mittels uuttu 'Nistkasten' steht bei T. I. Itkonen, Suomen lappalaiset vuoteen 1945 II. Porvoo-Helsinki 1948 S. 67-68. 


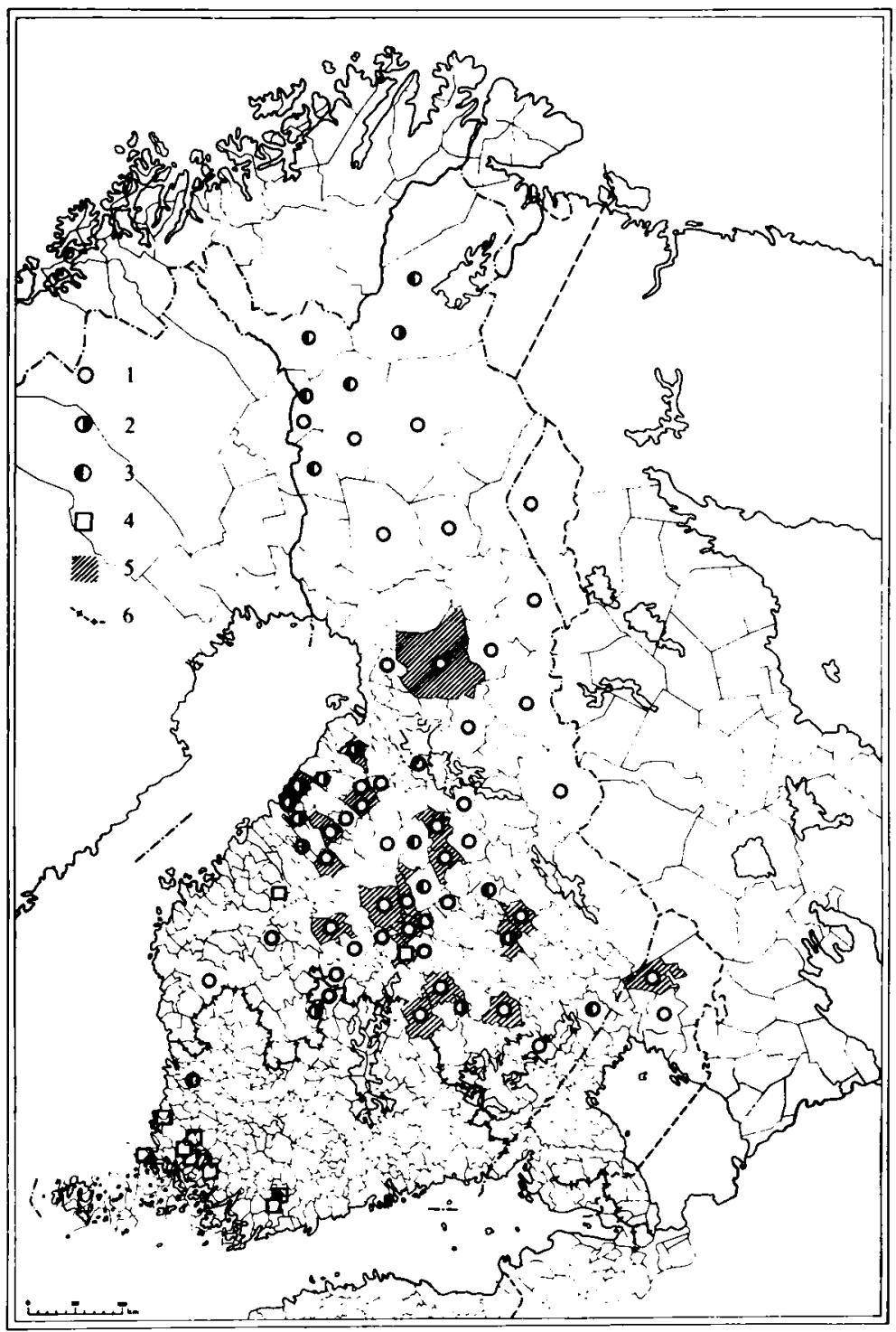

Karte 3

1 uuttu 'Nistkasten von Wasservögeln; Höhle im Baum; Birkhuhn- (Waldvogel-)Grube im Schnee'; 2 uutto = uuttu (1); $3 u u$ = uuttu (1); auch übertragen 'uusta ja ahtaasta' (Kiur PieV Nil), ukko uussa, parta jiässä (Kite) usw.; 4 uukku (uukko, Kus) 'Nistkasten' (Kus), 'Höhle (Lappa); 'Höhle, wo Krebse wohnen' (Myn Vaht Nou KarTl Lie PyhM); 'Schlafplatz des Waldvogels' (Konn Sam); 5 uuttu, uutto, uu, uukko 'Birkhuhn-(Waldvogel-), Hasen-Grube im Schnee'. 6 Nordgrenze von kieppi in den Bedeutungen 1-3 nach Karte 2 im 
mit den Jagdfahrten der Finnen in die Einöden in Anspruch genommen wurden. Dagegen konnte sich der unbedeutende Handel der Häme-Bewohner, der in dieser Zeit mit der Gegend am Finnischen Meerbusen stattfand, nicht mit jenen Handelsinteressen messen, die Nowgorod in immer stärkerem Masse durch bzw. über die Karelier bekundete. Pelzwerk und Tierhäute gehörten zu den wichtigsten Produkten. Die Wasserstrassen in Richtung Ladogasee stärkten die Stellung der Karelier im Osten.

Wir fragen uns nun, ob der veränderte Kultureinfluss aus dem Osten, dem die Lappen seit ca. dem 12. Jh. ausgesetzt waren, eine Erklärung sein kann für die Veränderungen in der Verbreitung und Bedeutung des Wortes, wie wir sie festgestellt haben. Es wäre verlockend, wie angedeutet, die Bedeutung von kieppi ${ }^{2}$ als ein Resultat östlichen Einflusses zu sehen.

Es gibt auch eine Stütze für die Annahme, und zwar in Verbindung mit den Veränderungen im Handel, die wir als eine Folge des Auftretens der Karelier im Osten kennen. ${ }^{67}$

Der Ausgangspunkt ist, dass die Nachfrage nach bestimmten Waren grösser ist als früher. Dass Tierfelle bzw. Lederprodukte stark gefragt waren, ist bekannt. Effizient konnte die Jagd nur von den Lappen ausgeübt werden. Aber auch in der Behandlung von Tierhäuten waren die Lappen so sachkundig und geschickt, dass gegerbte Häute, Leinen, Riemen, Schuhe udgl. aus Rentierleder gängige Produkte waren. Schon in dem Bericht über Other von Hålogaland, der aller Wahrscheinlichkeit nach in der Nähe des heutigen Tromsö ansässig war, wird davon berichtet,

${ }^{67} \mathrm{Zu}$ beachten ist der Unterschied, der noch heute in der Auffassung der Lappen darüber herrscht, wer ein Quäne ist (d.h. ein Westfinne in der alten Zeit; IpL gái'nolaš Kai'nōlatjD und was das Kennzeichen eines Kareliers ausmacht (lpL gár'jel karjēel D. Ersterer ist ein (hinzugezogener) Nichtlappe, der ein friedliches Leben als Bauer führt, während letzterer ein gefürchteter Eindringling ist, der in alter Zeit die Lappen verfolgte. In lappischen Sagen ist davon die Rede, wie diese Karelier in kleinen Gruppen die lappischen Gegenden auf- und heimsuchten. Hierdurch wird unser Wissen von den intensiven Handels- und Besteuerungsaktivitäten der Karelier gut veranschaulich. Vgl. zu der Stammesbezeichnung im Lappischen: Erkki Itkonen, Zwei Andenken an die Zeit von Karelien und Haalogaland. MSFOu 145. Helsinki 1968. 
dass die Lappen Steuern zahlten in Form u.a. von Rentierhäuten und Stricken aus Robben- und Walfischhaut. ${ }^{68}$ Die Herstellung von Leinen für den Hausgebrauch aus bearbeiteter Rentierhaut ist unter den Lappen in allen Gebieten bekannt.

Ein lappisches Lehnwort ${ }^{69}$, das den Wert der lappischen Lederbereitung und -verarbeitung zeigt, ist fi. siisna sisna 'Riemen aus Leder, Birkenrinde, Stoff etc.' (IpN sis'te 'tanned reindeer skin', K. Nielsen; IpL sas'nē 'Sämischleder', $H$. Grundström). Die Verbreitung des Wortes, die aus der Karte 4 hervorgeht, ist eindeutig ostfinnisch. Ausser den Belegen der Wörterbuchstiftung ist die von Valonen ${ }^{70}$ nachgewiesene Bedeutung 'Riemen aus Birkenrinde' mit aufgenommen worden. T. I. Itkonen registriert die südliche Verbreitung des Wortes in der letztgenannten Bedeutung mit Verwundern, da die Lappen ja keine Arbeiten aus Birkenrinde anfertigten. ${ }^{71}$

Die Entlehnung findet jedoch keine Erklärung, falls sie keine Funktion als Bezeichnung für ein typisch lappisches Produkt gehabt hat, und zwar Riemen oder Seile aus bearbeiteter Rentierhaut. Nur im höchsten Norden kann das finnische Wort eine ganze Rentierhaut bezeichnen.

Die fertigen Riemen oder Seile hat man begreiflicherweise irgendwie zusammengerollt verwahrt und transportiert. Es kann eine grosse Ähnlichkeit bestanden haben zwischen diesen zusammengerollten Lederriemen und den Birkenrindenriemen, die die Savoer herstellten. Es scheint durchaus glaubwürdig zu sein, dass wir gerade in diesem Punkt die Lösung dafür finden, warum sich siisna öfter auf Birkenrinde denn auf Leder bezieht. Ausserdem bietet sich hier auch eine Lösung unseres Problems mit der Bedeutung von kieppi ${ }^{2}$ an. Valonen erwähnt nämlich, dass die Rollen der fertigen, am Rand abgeschnittenen Birken-

${ }^{68}$ Björn Collinder, Lapparna 1953 S. 29.

${ }^{69}$ Frans Äimä, JSFOu XXV, I S. 38. Lappisch (lpN) sis'te und (lpL) sas'nē sind baltische Lehnwörter, die auch ihre Entsprechung im Finn. haben. Der Entlehnungsvorgang ist also: fi. hihna $<{ }^{*}$ šišna $>$ lp. sis'te $\sim$ sas'ne etc. $>$ fi. siisna sisna.

${ }^{70}$ Niilo Valonen, Geflechte und andere Arbeiten aus Birkenrindenstreifen. Kansatieteellinen Arkisto IX, Vammala 1952 S. 12-.

${ }^{71}$ T. I. Itkonen, Tuohi kansanomaisessa tekniikassa. Virittäjä 1953 S. 136. 


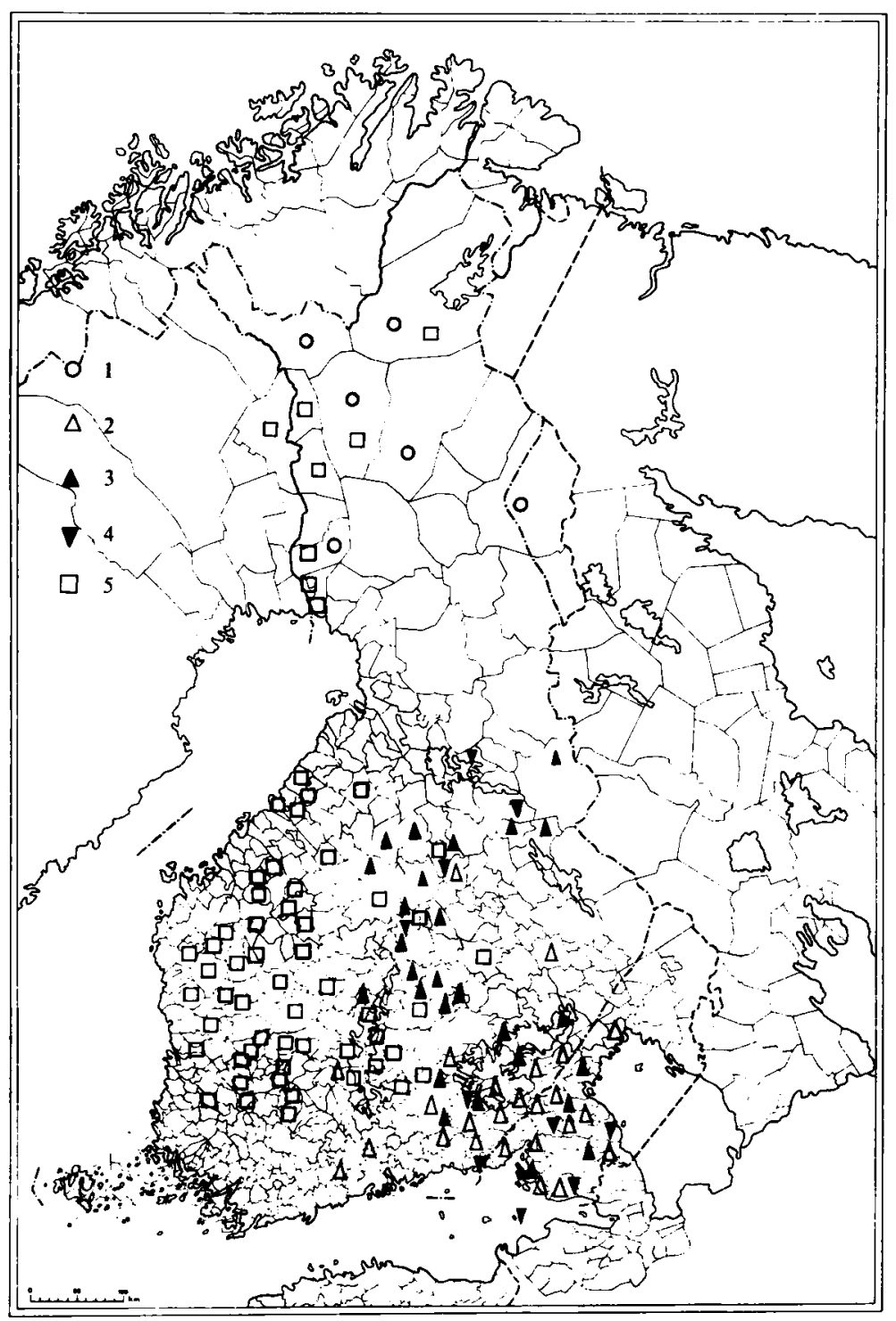

Karte 4

1 sisna (Äimä) 'geflochtenes Leder, Fell, dem das Haar ausgegangen ist'; (SS) 'schlechtes oder nicht gegerbtes Rentierleder; haarlos' (YTor); 2 siisna ( sisna) 'Streifen, Riemen, Band'; 3 siisna ( sisna) 'Birkenrindenstreifen, -band'; siiskale id. (KanN); siisla id. (Lemi); 4 siisna ( sisna)' Lederstreifen, Band'. 5 iltti 'Zunge, Lasche; Ober- od. Vorderleder von Schuhzeug'. 
rindenriemen, die so aufbewahrt oder verkauft werden sollten, kieppi hiessen (S. 15; s. Abb. S. 18). Eine solche Rolle konnte lang aufbewahrt werden und wurde erst zum Zwecke der Anwendung geöffnet. Aus der Abbildung geht hervor, dass die Rolle die Form eines Knäuels hatte, in dem eine kleine Öffnung gelassen wurde. Wer eine Übernachtungsgrube des Birkhuhns im Schnee mit dem kleinen Atemloch gesehen hat, kann das Aussehen einer solcher Birkenrindenrolle leicht mit der Grube eines Waldvogels im Schnee verbinden. Eine ähnliche Form hat auch ein Schnurknäuel oder eine zusammengekringelte Schlange. Diese Bedeutungen kommen auch von kieppi ${ }^{2}$ vor.

Kieppu hat dieselbe Bedeutung wie kieppi ${ }^{2}$, tritt aber (ausser in Ruov) in einem begrenzten Gebiet auf, das mit den Stellen der alten Lappendörfer zusammenfällt, die Vilkuna lokalisiert hat. Die Variante mit $-u$ kann nicht ohne Schwierigkeit als direkte Entlehnung aus dem Lappischen angesehen werden. ${ }^{72}$

Wir haben also festgestellt, dass die lappischen Lehnwörter südlich der heutigen lappischen Ansiedlungen eine erneuerte Funktion - auf die eine oder andere Weise herleitbar aus der alten - benötigen, um in der Sprache bewahrt werden zu können. Ein wesentlicher Teil der Identifikation des Wortes liegt in der Aufspürung der Ursachen für diese veränderte Funktion.

10. Die Geschicklichkeit der Lappen in der Behandlung von Leder und der Herstellung von Lederprodukten, z.B. Schuhen, kann durch ein anderes Wort, das von einigen Forschern als lappisches Lehnwort im Finnischen betrachtet wird, illustriert werden. Fi iltti 'Zunge, Lasche; Ober- od. Vorderleder von Schuhzeug' (Lönnrot) ist in dem Fall eine frühe Entlehnung ${ }^{73}$ aus (lpN) aldâs 'the upper piece of the front part of a Lapp shoe, from the tip to the top edge of the shoe (in front)' (K. Nielsen). Die Verbreitung des Wortes ist nicht derart abgegrenzt wie bei den oben angeführten Beispielen, sie kann aber trotzdem als

${ }^{72} \mathrm{Zu}$ bestimmten Ausnahmen bei den Vokalen der zweiten Silbe in Lehnwörtern s.: Frans Äimä, JSFOu XXV, 1 S. 53. Vgl. Nr. 65 oben.

${ }^{73}$ Frans Äimä, JSFOu XXV, 1 S. 54. SKES verzeichnet das Wort in Band I als Erbwort und mit Vorbehalt als Entlehnung im Finn. 


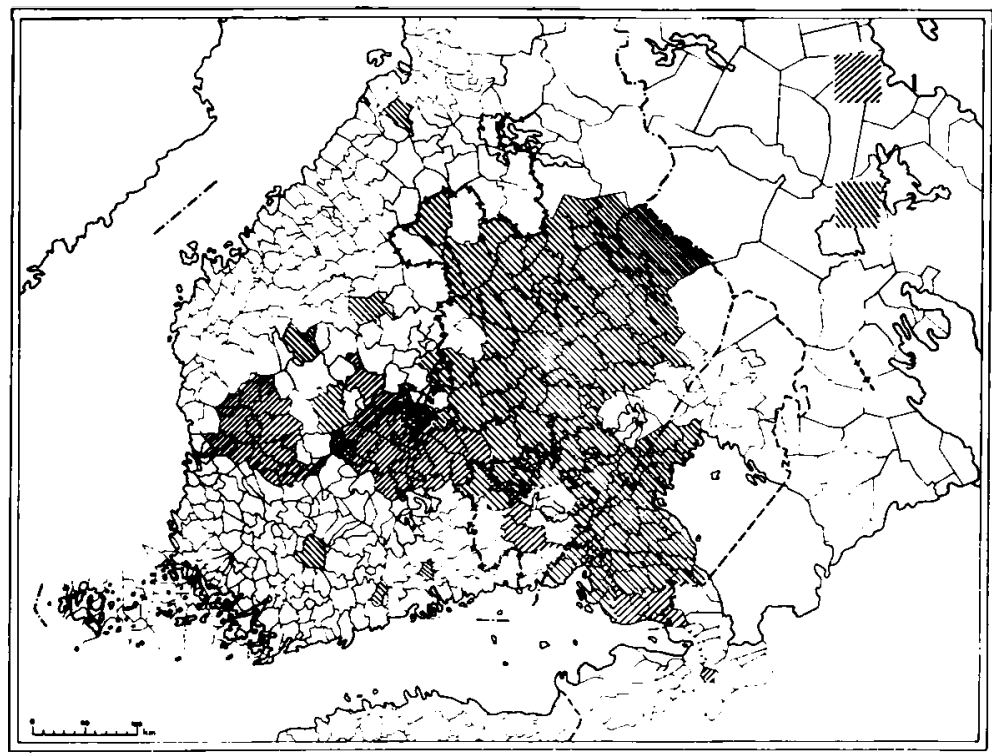

Karte 5

I kieppi in den Bedeutungen 1-3 nach Karte 2 im südlichen Finnland; 2 kieppi in den Bedeutungen 4-6; 3 Westgrenze von siisna sisna in den Bedeutungen 3-4 nach Karte 4.

westfinnisch charakterisiert werden. Beachtenswert ist die Ausbreitung im Süden, die ähnlich wie bei kurmu und kieppi die nördlichen Teile von Satakunta und Häme erreicht. Einige sporadische Belege finden sich im westlichen Savo.

Iltti ist ein Beispiel für ein Wort, das nicht festgelegt ist durch eine begrenzte Funktion, wodurch es an einen gewissen Umkreis gebunden wäre, sondern es spiegelt in seiner Gesamtheit die westfinnische Einödkultur wider, wozu auch die Kolonisierung des Tal des Torneälv (fi. Tornionjoki) gehört (s. die Belege am Torneälv).

Eine gründlichere Ermittlung könnte sicher jene Funktion ergründen, durch die diese Entlehnung motiviert war. Die Form der Schuhe kann - zusätzlich zu den Traditionen des Schuhnähens bei den Lappen - eine Ursache gewesen sein, denn darin liegt eine lappische Novation in der finnischen Kultur. 
11. Wie können wir nun unsere Darstellung vom Verhalten der genannten Lehnwörter zur frühen finnischen Einödkultur und Bevölkerungsexpansion mit dem Faktum vereinen, dass sowohl kurmu als auch kieppi in den Kirchspielen um die Grenze zwischen Uusimaa und Varsinais-Suomi begegnen? (Kurmu 'Kuh' Pai Kiika, 'magerer Mensch' Muurl Somero, 'elender Mensch' Kiika and kieppi in der Bedeutung 'Grube eines Waldvogels, Birkhuhns im Schnee' Tam.) Eine Mitteilung aus Tammela berichtet über die Birkhuhnjagd mit Fackeln. Kieppi ist "die Schlafgrube eines Vogels unter dem Schnee, vor allem die Birkhühner und Haselhühner schlafen da, 'mit dem Vater passten wir auf, wohin die Birkhühner zum Übernachten gehen und dann gingen wir auf Jagd' (lährettīn tülastammān)." (SS; Tammela, Vilho Viksten 1959.)

Wie ich bereits andeutete, bezeugt der Reichtum an lappischen Ortsnamen, dass hier noch in historischer Zeit Lappen wohnten. Voionmaas Untersuchung hat genauer dokumentiertes Material hierüber bieten können. ${ }^{74} \mathrm{Wie}$ in Obersatakunta hat sicher die späte Kolonisierung des Gebietes durch finnische Bevölkerung den Lappen die Möglichkeit des Überlebens gegeben. Es gibt schriftliche Angaben über die Lappen in der Gegend, durch die erklärt werden kann, warum gerade die Kirchspiele Tammela-Somero-Somerniemi-Kiikala eine so grosse Anzahl von Ortsnamen aufweisen, die mit Lappi-beginnen. Auch können wir sehen, dass kurmu und kieppi ihre südlichsten Belege hier haben. Voionmaa erwähnt nämlich eine Angabe von ca. 1492 über einen Bettler namens Eskeli Lappi (Eskillus Lappe), den der Pfarrer von Somero laut Beschwerde der Gemeindemitglieder schlimm behandelt habe. Dieser Name kann so gedeutet werden, dass sein Träger ein Lappe war. Åkerblom ${ }^{75}$ gibt eine alternative Deutung des Lapp(i)-Namens, der sowohl als Orts- wie als Familienname im südwestlichen Finnland zurückgehen könnte auf den Wortstamm in fi. laper-

\footnotetext{
74 Väinö Voionmaa 1943 (s. Anm. 7).

${ }^{75}$ Bror Åkerblom, Lapp-namnen i Finland. Folkmålsstudier XI. Helsingfors 1945.
} 
taa 'prahlen' (S. 27) und lepertää 'schwatzen, schmeicheln' (S. 29). Ein solcher Name könne somit nicht mit Sicherheit auf lappische Bevölkerung hinweisen. Dennoch ist der Verfasser auch der Meinung, dass "ein Teil der Lapp-Namen in Uusimaa und in der Umgebung des heutigen Turku ( $\AA$ bo) Andenken enthält an die Streifzüge des lappischen Volkes und ihre Teilnahme am Handel vor mehreren hundert Jahren" (S. 51).

Mit Hilfe einer engeren Zusammenarbeit zwischen den verschiedenen Wissenschaftsdisziplinen wie z.B. Geschichte, Ethnographie, Archäologie und Sprachwissenschaft sollte es möglich sein, eine grössere Kenntnis der Lebensbedingungen und der Verbreitung der lappischen Bevölkerung in alter Zeit zu erhalten. Dieser Artikel hat die diesbezüglichen Möglichkeiten aufzeigen wollen.

OlaVi KORHONEN

Abkürzungen der Kirchspiel-Namen:

$\begin{array}{llll}\text { Avus } & \text { Alavus } & \text { Kih } & \text { Kihniö } \\ \text { EnoT } & \text { Enontekiö } & \text { Kiika } & \text { Kiikala } \\ \text { Erä } & \text { Eräjärvi } & \text { Kite } & \text { Kitee } \\ \text { Hir } & \text { Hirvensalmi } & \text { Kitt } & \text { Kittilä } \\ \text { HKyr } & \text { Hämeenkyrö } & \text { Kiur } & \text { Kiuruvesi } \\ \text { HonJ } & \text { Hongonjoki } & \text { Kive } & \text { Kivennapa } \\ \text { Ika } & \text { Ikaalinen } & \text { Kong } & \text { Konginkangas } \\ \text { Ilm } & \text { Ilmajoki } & \text { Konn } & \text { Konnevesi } \\ \text { Jal } & \text { Jalasjärvi } & \text { KorL } & \text { Korpilahti } \\ \text { Jur } & \text { Jurva } & \text { KosP } & \text { Koskenpää } \\ \text { Jämi } & \text { Jämijärvi } & \text { KuhL } & \text { Kuhmalahti } \\ \text { Jäms } & \text { Jämsä } & \text { KuoV } & \text { Kuorevesi } \\ \text { KanA } & \text { Kangasala } & \text { Kus } & \text { Kustavi } \\ \text { KanJ } & \text { Kanneljärvi } & \text { Kärs } & \text { Kärsämäki } \\ \text { KanN } & \text { Kangasniemi } & \text { Laih } & \text { Laihia } \\ \text { KanP } & \text { Kankaanpää } & \text { Lappa } & \text { Lappajärvi } \\ \text { Kars } & \text { Karstula } & \text { Lapu } & \text { Lapua } \\ \text { KarTl } & \text { Karjala T1 } & \text { Lie } & \text { Lieto } \\ \text { Karv } & \text { Karvia } & \text { Luu } & \text { Luumäki } \\ \text { KauJ } & \text { Kauhajoki } & \text { Län } & \text { Längelmäki } \\ \text { KemJ } & \text { Kemijärvi } & \text { Mes } & \text { Messukylä } \\ \text { Keu } & \text { Keuruu } & \text { Mik } & \text { Mikkeli }\end{array}$




$\begin{array}{llll}\text { Muon } & \text { Muonio } & \text { SavT } & \text { Savitaipale } \\ \text { Muurl } & \text { Muurla } & \text { Siikai } & \text { Siikainen } \\ \text { Myn } & \text { Mynämäki } & \text { Sip } & \text { Sippola } \\ \text { Nil } & \text { Nilsiä } & \text { Sod } & \text { Sodankylä } \\ \text { Nmo } & \text { Nurmo } & \text { Suoni } & \text { Suoniemi } \\ \text { Nou } & \text { Nousiainen } & \text { Sys } & \text { Sysmä } \\ \text { Oriv } & \text { Orivesi } & \text { Tam } & \text { Tammela } \\ \text { Pad } & \text { Padasjoki } & \text { TuuN } & \text { Tuusniemi } \\ \text { Pai } & \text { Paimio } & \text { Töy } & \text { Töysä } \\ \text { Park } & \text { Parkano } & \text { UusVl } & \text { Uusikirkko Vpl } \\ \text { PerS } & \text { Peräseinäjoki } & \text { Vaht } & \text { Vahto } \\ \text { PieV } & \text { Pielavesi } & \text { Vesa } & \text { Vesanto } \\ \text { Pom } & \text { Pomarkku } & \text { VesL } & \text { Vesilahti } \\ \text { PyhM } & \text { Pyhämaa } & \text { Viit } & \text { Viitasaari } \\ \text { PyhOl } & \text { Pyhäjärvi Ol } & \text { Vilj } & \text { Viljakkala } \\ \text { Sah } & \text { Sahalahti } & \text { Vär } & \text { Värtsilä } \\ \text { Sall } & \text { Salla } & \text { YTor } & \text { Ylitornio } \\ \text { Sam } & \text { Sammatti } & & \end{array}$

